\title{
The development of an intervention package to prevent children under five years old drowning in rural Bangladesh
}

\begin{abstract}
Aim:

There are an estimated 372000 worldwide deaths by drowning every year, and it has been described as a secret epidemic in Bangladesh. The aim of this study was to develop an intervention package to prevent children under the age of five from drowning in rural Bangladesh.

\section{Methods:}

This was a qualitative study using focus group discussions in three villages in rural Bangladesh. The 45 participants were mothers and fathers with children under five, the parents of children who had drowned and community leaders.

\section{Results:}

The majority of the participants $(71 \%)$ were male. The focus groups revealed that most drowning's occurred between $11 \mathrm{am}$ and $2 \mathrm{pm}$ and that risk factors included the following: children not being able to swim, ditches that were not filled in, lack of medical facilities, parents who were not aware of childhood drowning and lack of information through the media about how to prevent of childhood drowning. Suggestions included using a mobile-based short messaging service or voice calls to parents, especially mothers, could increase awareness and reduce the risk of childhood drowning.
\end{abstract}

\section{Conclusion:}

A safety education programme could be effective in increasing knowledge and changing attitudes, which could prevent drowning among children in Bangladesh.

Keyword: Bangladesh; Childhood drowning; Mobile phones; Safety education; Short messaging service 